\title{
Fine Structure and Organization of the Sarcoplasmic Reticulum in the Sino-Auricular Fibres of Frog Heart
}

\author{
Michel Duvert and André L. Barets \\ Laboratoire de Cytologie, Université de Bordeaux 2, Avenue des Facultés, F-33405 Talence- \\ Cedex-France
}

Z. Naturforsch. 34 c, 865 - 875 (1979); received May 25, 1979

Cardiac Muscle, Excitation-Contraction Coupling, Sarcoplasmic Reticulum, In Situ SR Morphology, SR-Mitochondria Relationship

The ultrastructure of the sarcoplasmic reticulum (SR) of the sino-auricular fibres of frog heart has been investigated especially in order to evaluate its possible relationship with several cellular organelles.

The SR appears as a complex unit which can be divided into three regions:

- peripheral SR, localized beneath the sarcolemma. It is composed of junctional SR, which forms specialized complexes (couplings) with the sarcolemma, and peripheral SR s. str. which frequently extends its anastomotic tubules around the caveolae.

- juxtafibrillar SR, in relation with myofibrils, at the level of $Z$ lines and I bands.

- intermediary SR which is the most abundant. It contains dense granules, able to bind strontium (and calcium) and, from place to place, is formed into flattened saccules bearing fenestrations where microfilaments frequently pass through. It allows peripheral SR and juxtafibrillar SR to communicate, giving unity to the reticular system and, on the other hand, shows close topographical relations with mitochondria.

The great extension of the SR, its heterogeneity and its relationships with several cellular organelles, especially mitochondria, are interpreted from the functional point of view.

\section{Introduction}

The possible participation of mitochondria in the control of calcium movements among the different cellular compartments which perform excitationcontraction coupling in striated muscle fibres is presently being seriously taken into consideration, particularly in the myocardium. It is based upon the ability of mitochondria to accumulate calcium by an active transport and to release it $[1,2]$. On a general point of view, the regulation of intracellular calcium by mitochondria has recently been reviewed by $\mathrm{Ca}$ rafoli and Crompton [3].

In Vertebrate myocardial fibres, two modes of action for mitochondria have been put forward:

(1) Mitochondria, in association with the sarcoplasmic reticulum (SR) and the plasma membrane, would participate in the regulation of sarcoplasmic calcium concentration at each cycle of contraction [4-8]. This hypothesis is questioned by Scarpa and Graziotti [9], Vinogradov and Scarpa [10] and by Kitazawa [11]. Schaffer et al. [12] agree with it in the case of frog heart but not in that or rat heart.

(2) Since their rate of calcium pumping is relatively slow in comparison with that of the SR,

Reprint requests to Dr. Michel Duvert.

0341-0382/79/0900-0865 \$01.00/0 mitochondria could not be active in the control of calcium movements at each cycle of contraction but could instead participate in regulating the steady state $\mathrm{Ca}^{2+}$ levels and thus the resting tension of the myofibrils [13, 14].

Most of these studies being of biochemical nature and performed with cell-free fractions, it seemed interesting to us to reexamine the whole morphological characteristics of the SR and its relations with various cellular organelles especially mitochondria, and to complete this study by the characterization of sites able to bind calcium.

For this study we selected the sino-auricular fibres of frog heart. They are easy to handle and they have become a reference material as shown by the numerous physiological studies devoted to them. Their mechanical activity has two components: phasic, induced by the entry in the cell of extracellular calcium (slow $\mathrm{Ca}$ inward current) and tonic, induced by the release of calcium out of some intracellular site, probably SR $[15,16]$. Another interesting characteristic is the lack of $\mathrm{T}$ system agreed on by almost all authors as well in the frog (auricular and ventricular muscles) as in the toad ventricle [17-23].

To describe the coupling zone between the SR and the sarcolemma we adopted the terminology proposed by Sommer and Johnson [19], the most widely used presently. 


\section{Materials and Methods}

Adult frogs (Rana esculenta) of both sexes were used.

(1) After pitching the spinal cord, the heart was removed, opened and immersed in a Ringer solution containing $\mathrm{MnCl}_{2}$ where its beat stopped. Fine sinoauricular trabecules were dissected out and put in the fixative. Best results were obtained with the Karnovsky fluid [24] in which aldehyde concentration was reduced by half. After a $2-3 \mathrm{~h}$ fixation at room temperature, the tissue fragments were washed in $0.2 \mathrm{M}$ cacodylate buffer and placed in $1 \% \mathrm{OsO}_{4}$ in $0.1 \mathrm{M}$ cacodylate buffer. Then they were stained with $1 \%$ aquous uranyl acetate, dehydrated in ethanol and embedded in Epon. Thin sections were stained with alcoholic uranyl acetate followed with lead citrate.

(2) The method of metallic staining developed by Thiéry and Rambourg [25] has also been used and gave a complete and regular staining of the SR and the mitochondria. As observed on relatively thick sections $(0.5 \mu \mathrm{m})$ a reasonably synthetic view of these structures can be obtained with this type of preparation.

(3) Sites able to bind calcium were visualized with the method described for the first time by Peachey [26] where calcium is replaced by strontium, an osmic fixation being necessary. The heart was removed, opened and pined in a Petri dish containing the following modified Ringer solution: $\mathrm{NaCl}$, $110.5 \mathrm{~mm} ; \mathrm{KCl}, 2.5 \mathrm{~mm} ; \mathrm{SrCl}_{2}, 18 \mathrm{~mm}$ (instead of $1.8 \mathrm{mM} \mathrm{CaCl}_{2}$ in normal Ringer); Tris- $\mathrm{HCl}, 10 \mathrm{~mm}$ (pH: 7.8). After $1 \mathrm{~h}$ incubation, heart beat was stopped with $\mathrm{MnCl}_{2}$; small pieces of tissue were dissected out and immersed $\left(4 \mathrm{~h}\right.$ at $\left.0{ }^{\circ} \mathrm{C}\right)$ in the fixative $\left(1 \% \mathrm{OsO}_{4}\right.$ in $0.15 \mathrm{M}$ cacodylate buffer containing $18 \mathrm{mM} \mathrm{SrCl}_{2}$ ); the tissue was washed in $0.15 \mathrm{M}$ cacodylate buffer containing $18 \mathrm{~mm} \mathrm{SrCl}_{2}$ dehydrated in ethanol and embedded in Epon. Sections were examined without any further staining.

\section{Results}

\section{Ultrastructure of the sarcoplasmic reticulum $(S R)$}

The SR is well developed in the sino-auricular fibres of frog heart. It is always abundant in the sarcoplasmic zones and shares this space in particular with mitochondria and specific granules of the myocardium.

From its localization in the muscle cell and its relations with one or the other cell organelles, three main regions can be distinguished.

\section{Peripheral sarcoplasmic reticulum}

It is situated beneath the sarcolemma and is composed of:

- the "junctional SR" which performs the "coupling" with the sarcolemma. The junctional SR membrane which faces the sarcolemma a) lies parallel to it, thus the distance between them (about $15 \mathrm{~nm}$ ) is constant, b) is flanked on its hyaloplasmic face, with dark structures, roughly triangular or hemispheric ("junctional processes"); these structures pointing towards the sarcolemma, are about $7.5 \mathrm{~nm}$ high and rather regularly spaced by a distance of $30 \mathrm{~nm}$. The cavity of the junctional SR, which can be more or less large, is characterized by the constant occurrence of "junctional granules" situated in a plan at a regular distance of about $7.5 \mathrm{~nm}$ from the SR membrane facing the sarcolemma (Figs 1, 2, and 3). Owing to the absence of transverse tubules $(=T$ system) in the sino-auricular fibres of the frog heart, couplings are localized on the sarcolemma.

- the peripheral SR s. str., a simple reticulum of tubules among which caveolae are frequently intermingled (Fig. 17).

The two types of peripheral SR as well as the SR situated more deeply in the cell, to be studied later, are all linked together (Figs 4, 5, and 17).

2. Intermediary sarcoplasmic reticulum

This name is given to the SR which extends between the peripheral SR, linked to the sarcolemma

Figs $1-20$. Sino-auricular fibres of frog heart.

Figs 1, 2, 3. "Couplings". Junctional sarcoplasmic reticulum (J-SR) containing "junctional granules" (JG) and coated towards the sarcolemma (S) with "junctional processes" (JP).

Fig. $1 \times 125000$; Fig. $2 \times 98000$; Fig. $3 \times 170000$.

Figs 4, 5. Continuity between the junctional sarcoplasmic reticulum (J-SR) and the intermediary sarcoplasmic reticulum (I-SR). The latter shows fenestrations where microfilaments can be seen (arrowheads). C, caveolae.

Fig. $4 \times 70$ 000; Fig. $5 \times 72000$. 


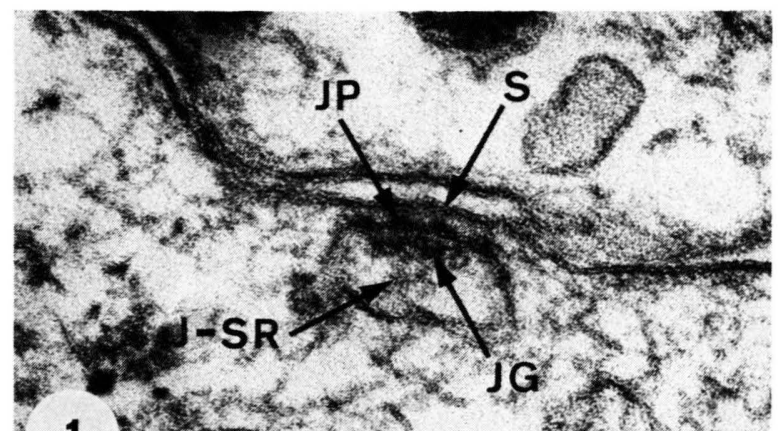

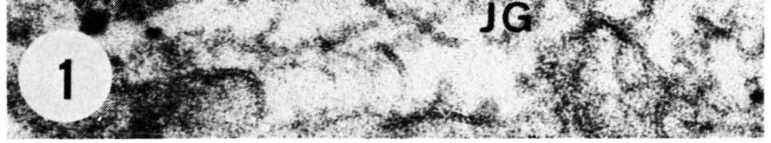
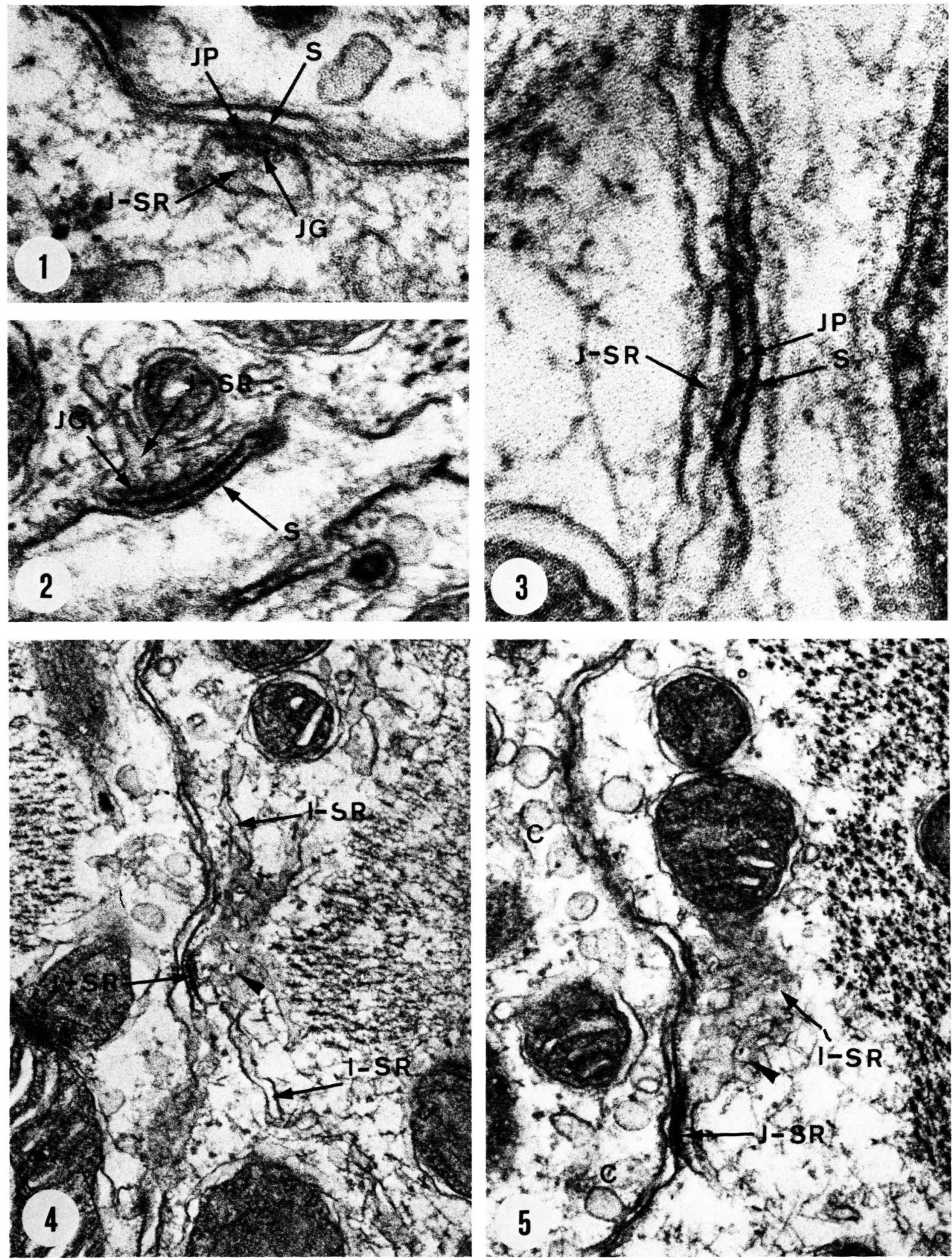

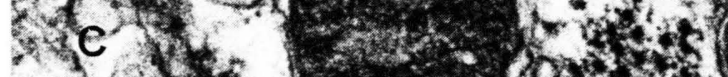

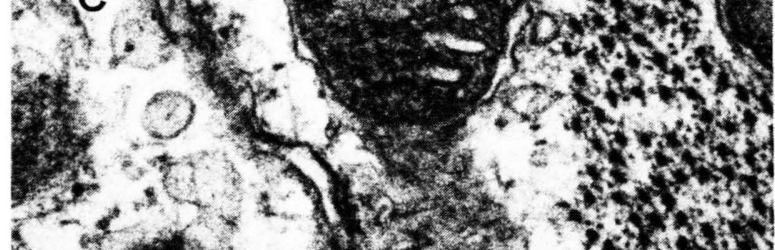

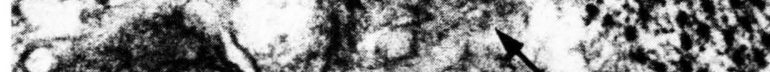

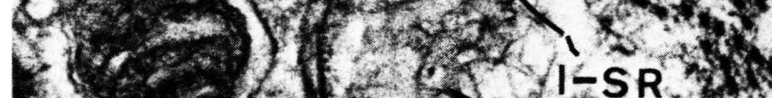

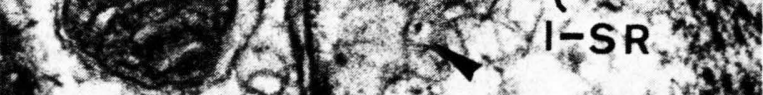

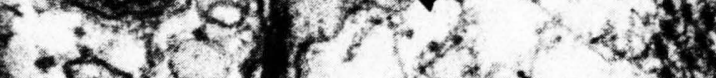

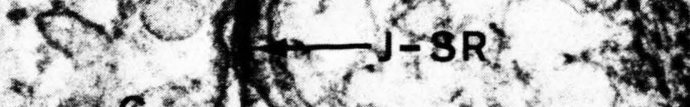

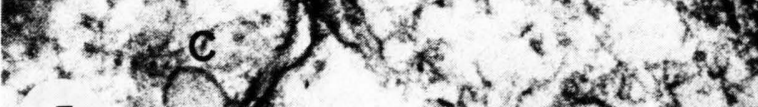
5 - 5 -

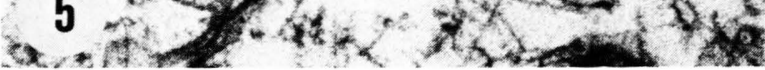



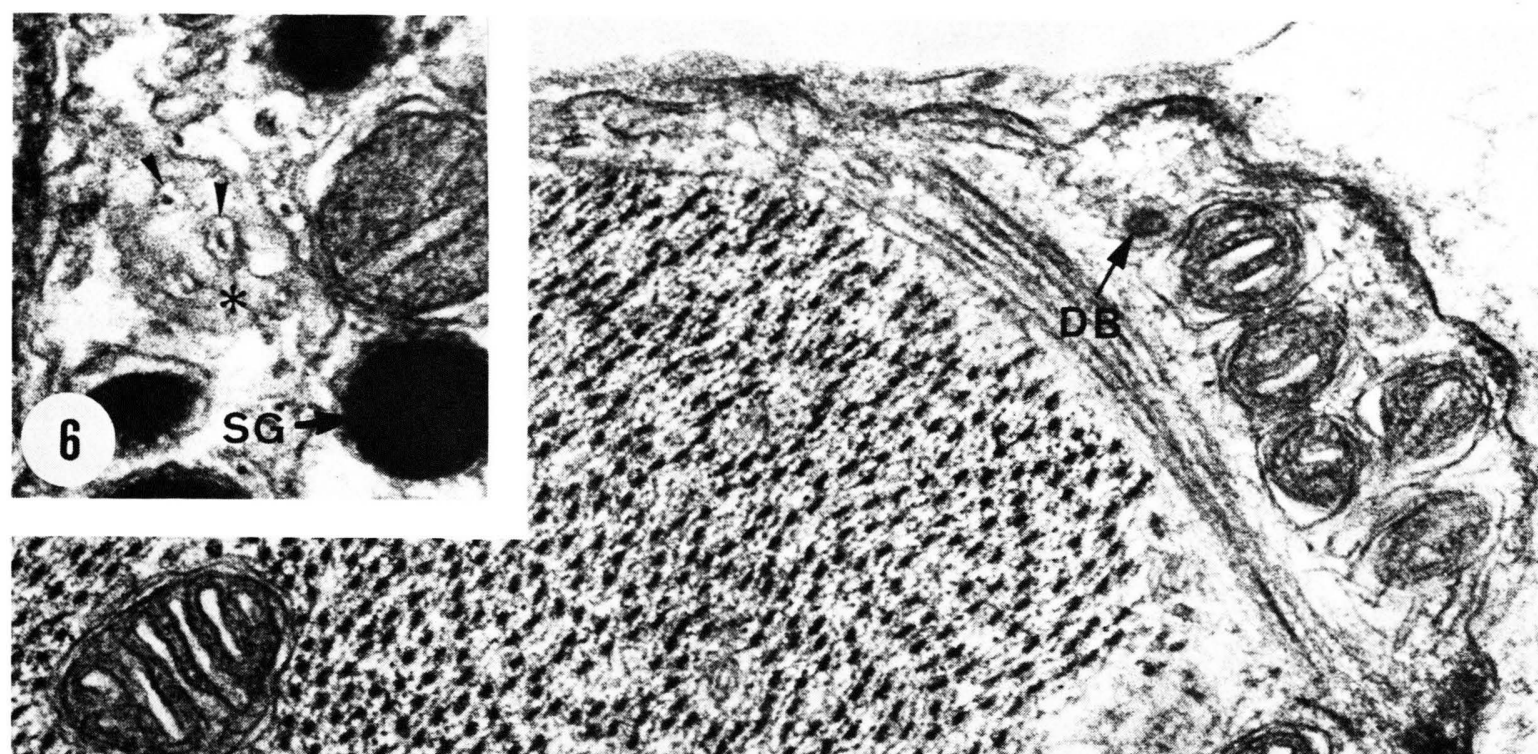

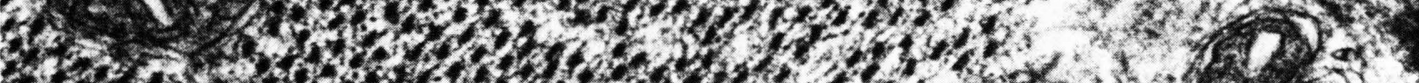

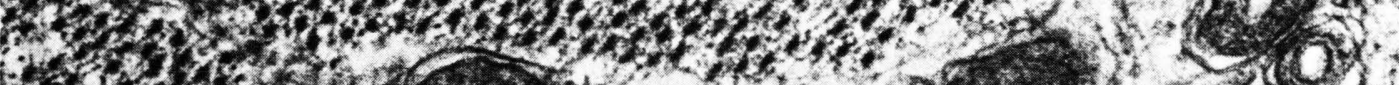
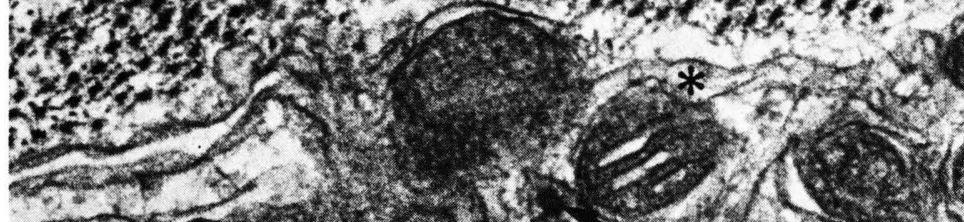

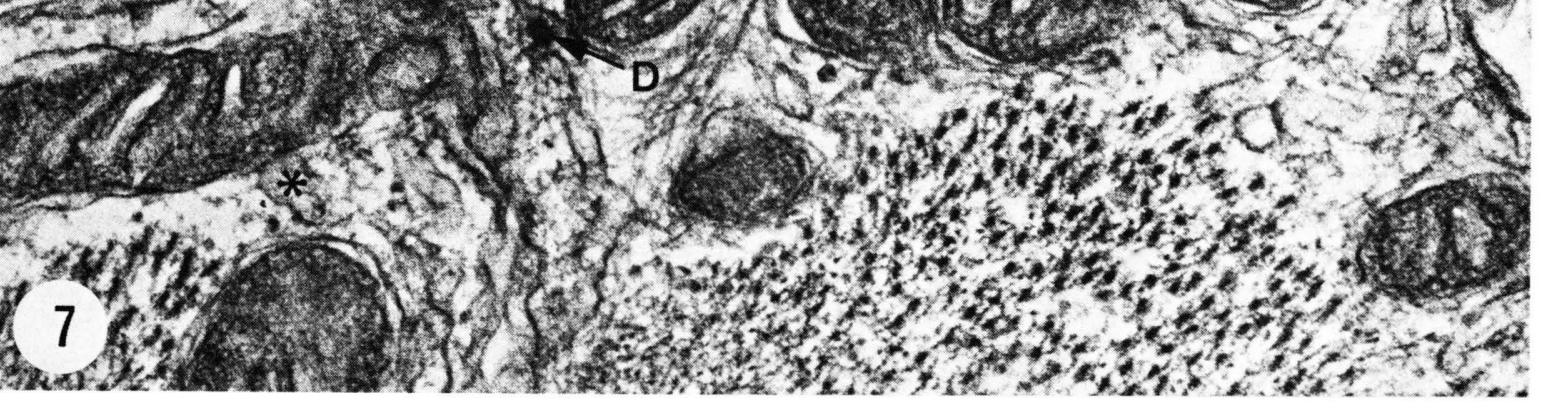

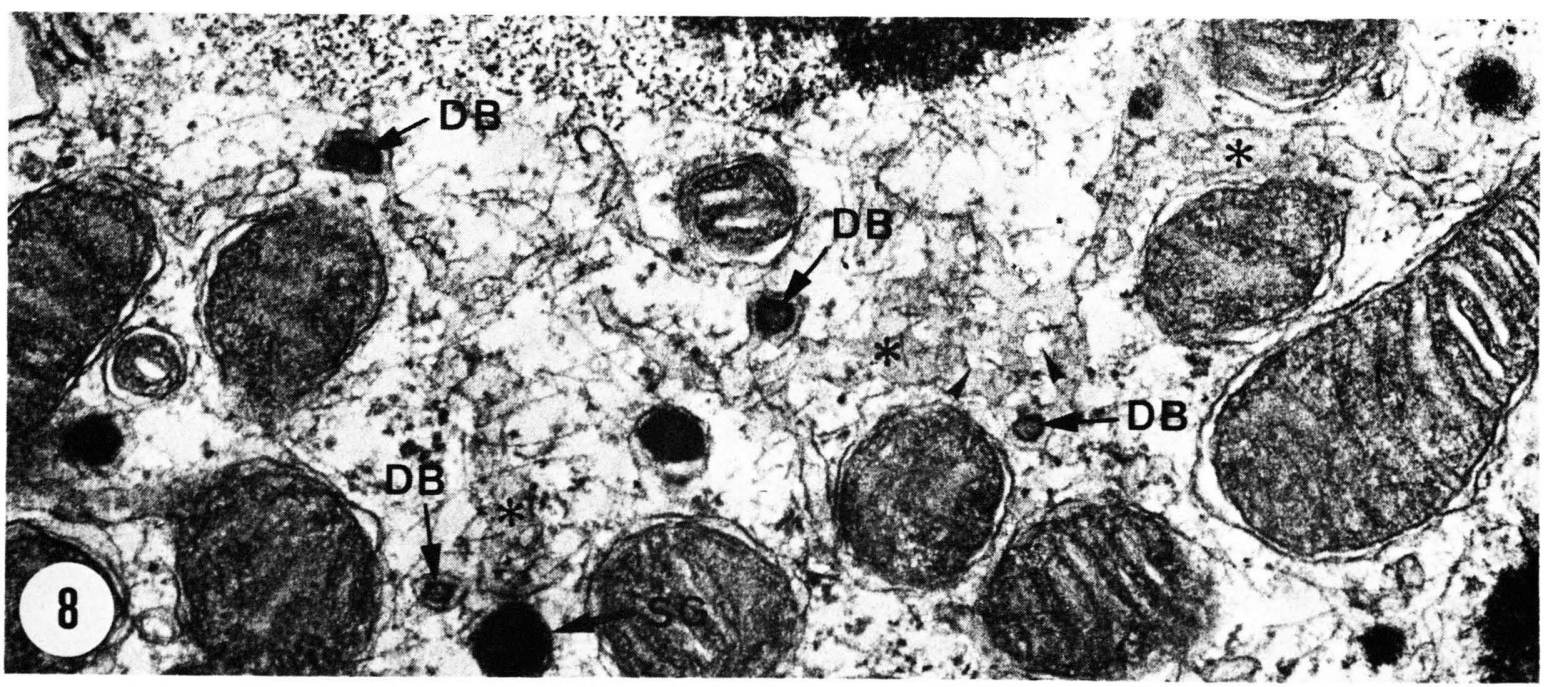


M. Duvert and A. L. Barets · Sarcoplasmic Reticulum in Frog Heart

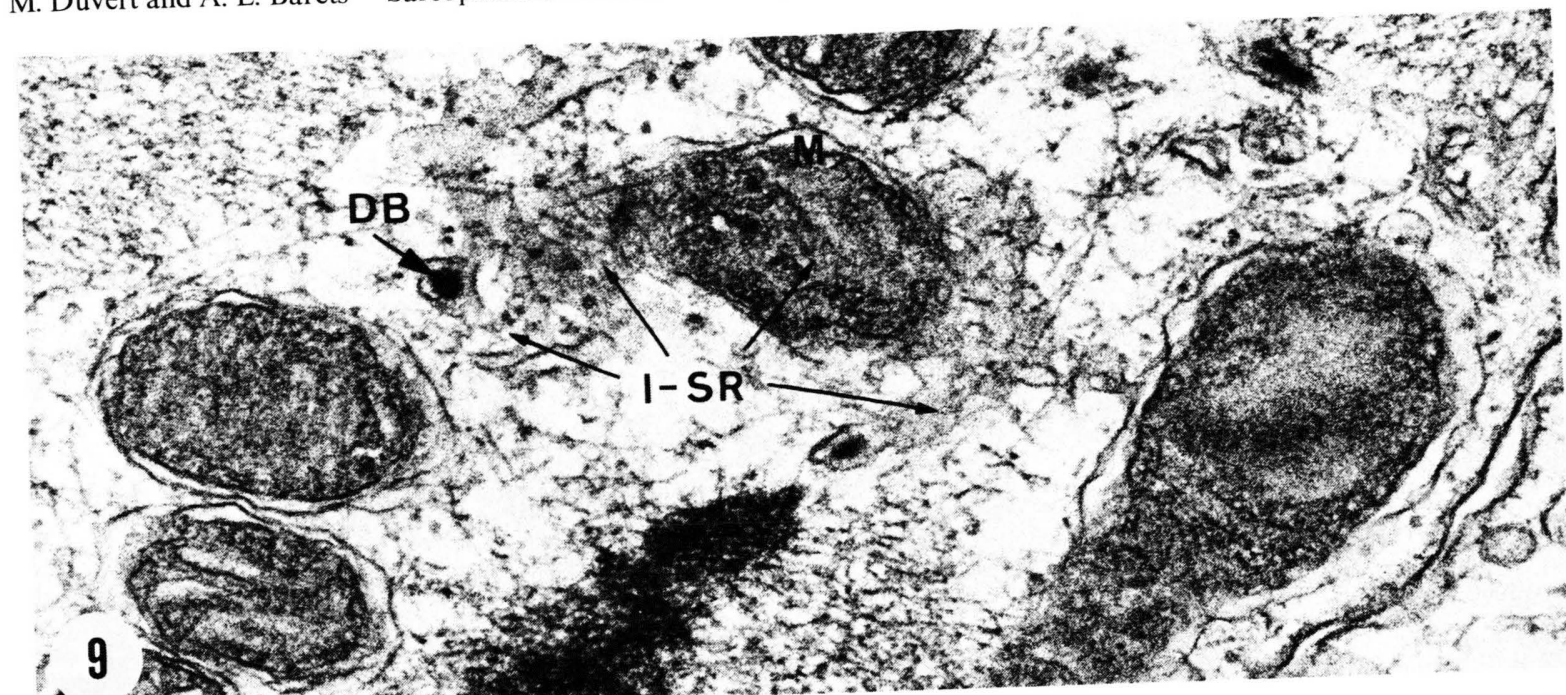

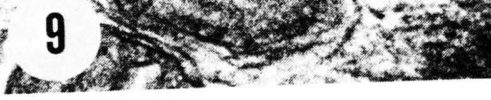

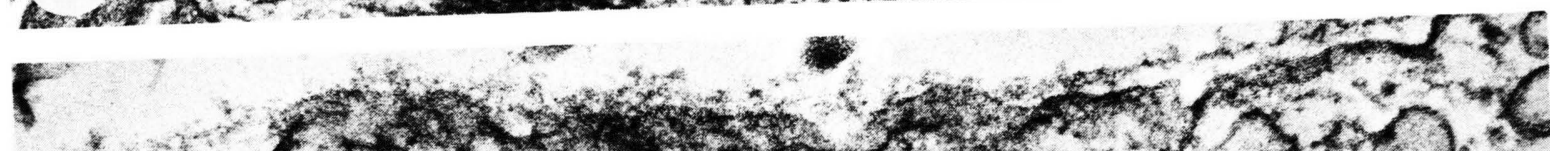
3
3

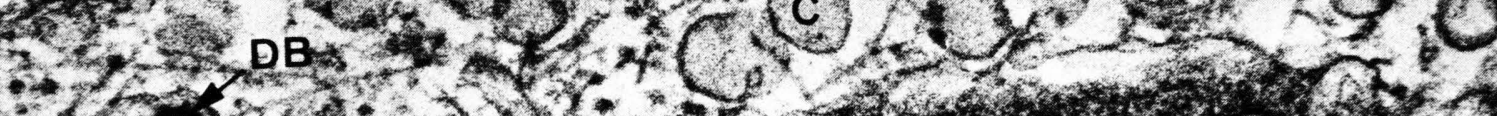

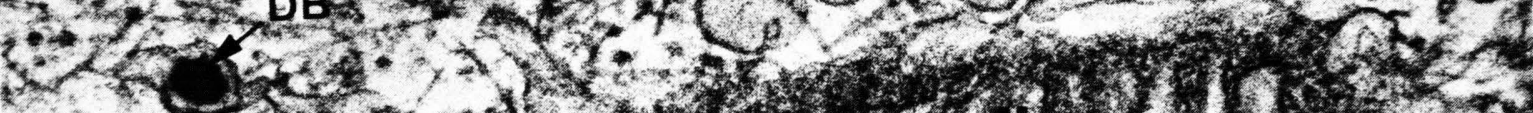

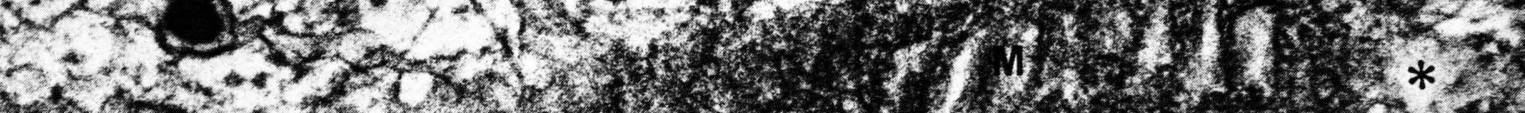

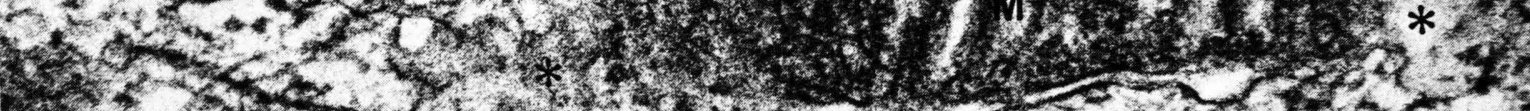

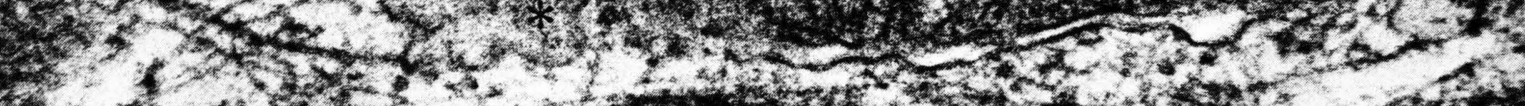

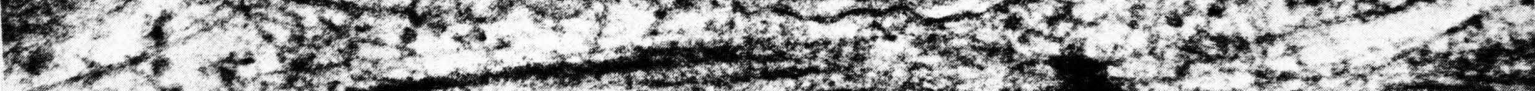

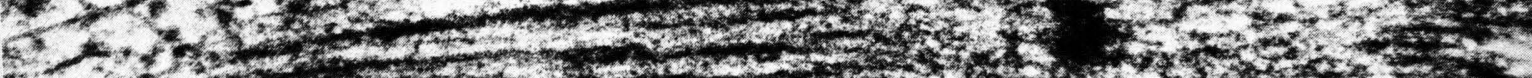

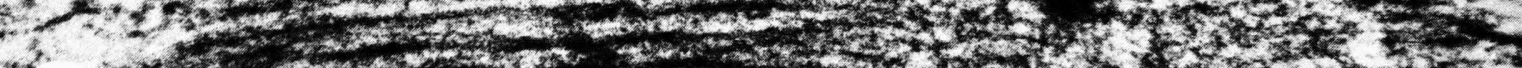

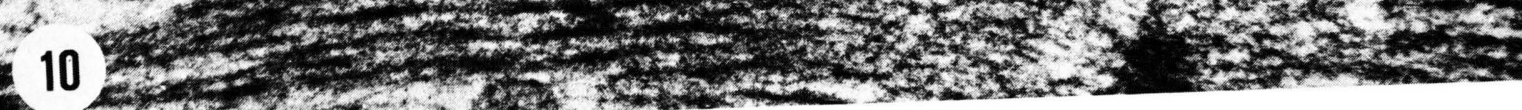

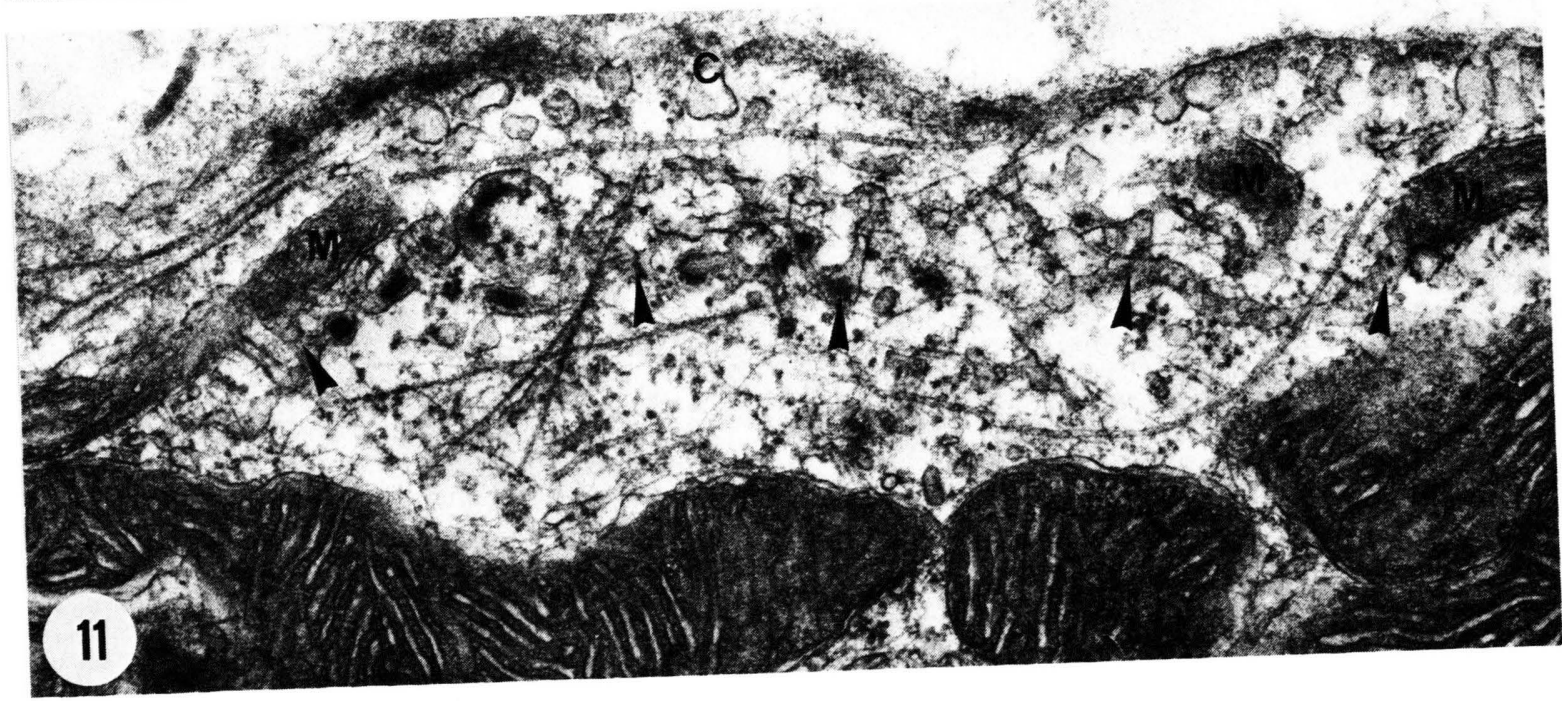


by its couplings on the one side and, on the other side, the SR associated with the myofibrils, as we shall see later and which will be called juxtafibrillar SR.

The intermediary SR represents the bulk of the entire SR of the cell. It looks as a more or less tight reticulum of tubules (Figs 7 and 8 ) which gives rise, from place to place, to large flattened saccules showing characteristic fenestrations in which microfilaments can be seen (Figs 4, 5, 6, and 8). In the cavity of the tubules occur dense bodies of variable shapes (spherical, ovoid or rodlike) with irregular contours and a core which often appears clear (Figs 2, 7, 8, 9, 10,11, $12,13,14$, and 15). The dimension of these dense bodies $(30$ to $70 \mathrm{~nm})$ makes it easy to distinguish between them and specific granules of the myocardium (100 to $200 \mathrm{~nm}$ ) (Figs 6 and 8). These latter show a regular spherical form and never possess a clear central core. The most interesting characteristic of the intermediary SR is, nevertheless, its close topographical relation with mitochondria.

At first glance it looks as if the external membrane of mitochondria is frequently more or less surrounded with SR profiles, but the relative exiguity of the volume available between the myofibrils makes the vicinity of the SR and of the mitochondria inevitable and not necessarily significant. Nevertheless, a more careful observation shows that relations between these two organelles are closer than those imposed by small volume. In sections tangential to mitochondria one can see that flattened saccules of the SR are actually apposed against the external membrane of the mitochondria since both organelles are superimposed over a relatively large area (Fig. 9). In other sections, slightly oblique viz mitochondria, when section leaves the mitochondria, it enters immediately the saccules or the tubules of the SR, tightly apposed to it (Figs 10 and 11). Detailed relations between the SR membrane and the mitochondrial external membrane cannot be analysed only by ultrastructural studies; for example, the apparent continuity observed between these membranes (Figs 12 and 13) may well be an artifact due to the obliquity of the section plan. It must be added that intra-reticular dense bodies seem to be particularly abundant in the juxta-mitochondrial SR (Fig. 11).

The region where intermediary SR and mitochondria are intermingled is particularly rich in microtubules and mostly in microfilaments (Figs 10 and 11). A microfilament is frequently observed in fenestrations of the flattened saccules of the SR (Fig. 6).

\section{Juxtafibrillar sarcoplasmic reticulum}

Some profiles of the SR show extremely close relations with the myofibrils at the level of the $\mathrm{Z}$ line (Figs 14 and 15). This "juxtafibrillar" reticulum is in continuity with the intermediary SR (Fig. 14).

\section{Metallic staining of the SR and of the mitochon- dria}

The method of Thiéry and Rambourg results in a very regular and complete staining of SR and mitochondria and shows the large development of the SR in inter-myofibrillar zones (Figs 16 and 17). One can also easily recognize, in section plans tangential to the sarcolemma, the reticulum of tubules of the peripheral SR, often surrounding caveolae, transver-

Figs $6,7,8$. Extension of the intermediary sarcoplasmic reticulum $(*)$. It can be formed into saccules with fenestrations where microfilaments pass through (arrowheads). DB, intra-reticular dense bodies, with irregular shape, often clear in their central core; SG, specific granules of the myocardium, spherical, larger than DB, never clear in their central core.

Fig. $6 \times 88$ 000; Fig. $7 \times 70000$; Fig. $8 \times 56000$ (s. p. 868 ).

Figs 9, 10. Topographical relations between intermediary sarcoplasmic reticulum and mitochondria.

Fig. 9. Section tangentiel to a mitochondria, showing superposition of the mitochondria (M) and of a saccule of the intermediary sarcoplasmic reticulum (I-SR) closely apposed to the former. DB, intra-reticular dense body. $\times 56000$

Fig. 10. Section slightly oblique viz a mitochondria, showing the passage from the mitochondria (M) to the intermediary sarcoplasmic reticulum $(*)$ which is closely apposed to it. C, caveolae; DB, intra-reticular dense body. $\times 75000$.

Fig. 11. Same situation. Arrowheads show the intermediary SR which appears as soon as the section plan leaves the mitochondria (M). Abundance of microtubules and of microfilaments can be noted. C, caveolae. $\times 44000$ (s. p. 869 ).

Figs 12, 13. Topographical relations between tubules of the intermediary sarcoplasmic reticulum containing dense bodies (DB) and the mitochondria external membrane (arrowheads).

Fig. $12 \times 102000$; Fig. $13 \times 60000$.

Figs 14,15 . Topographical relations between the juxtafibrillar sarcoplasmic reticulum $(*)$ and the myofibrils at the $Z$ level (arrowheads). The continuity of the JF-SR with the I-SR containing dense (DB) must be noted.

Fig. $14 \times 80000$; Fig. $15 \times 88000$. 
M. Duvert and A. L. Barets · Sarcoplasmic Reticulum in Frog Heart

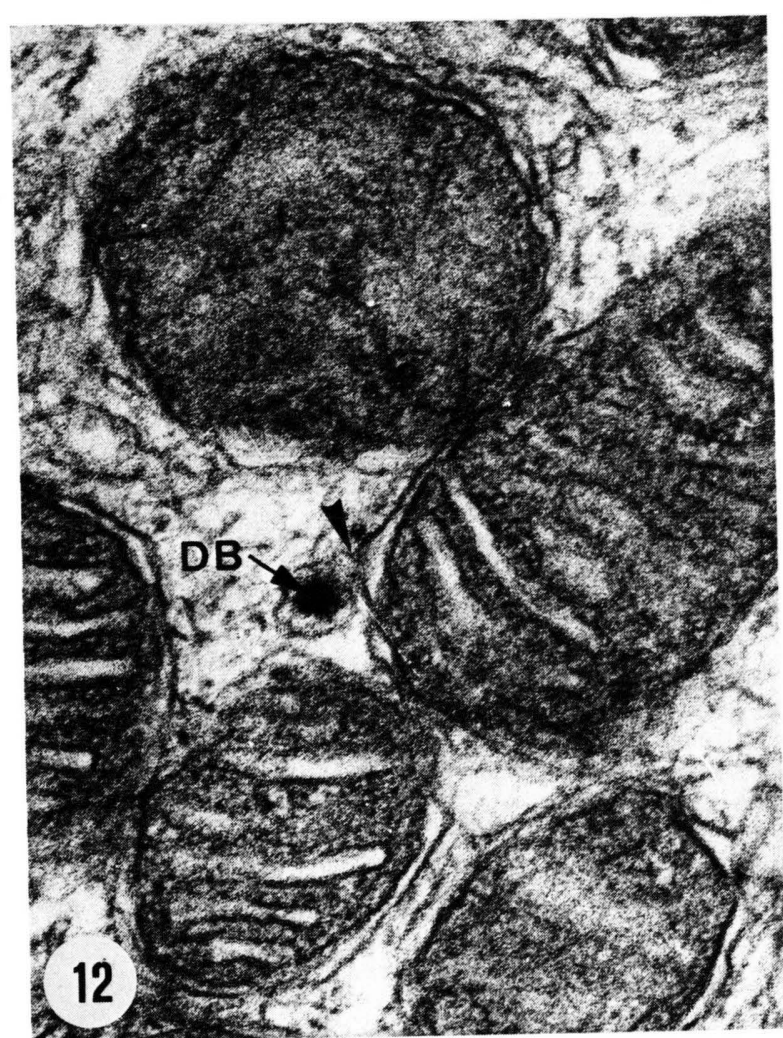

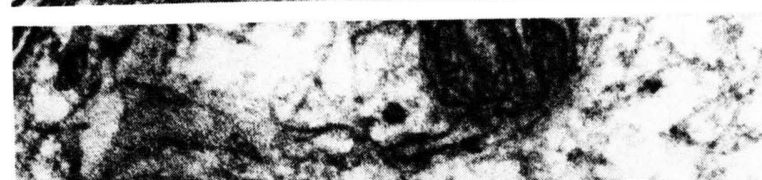

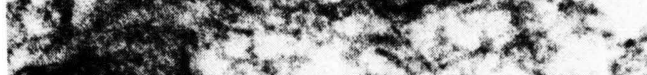

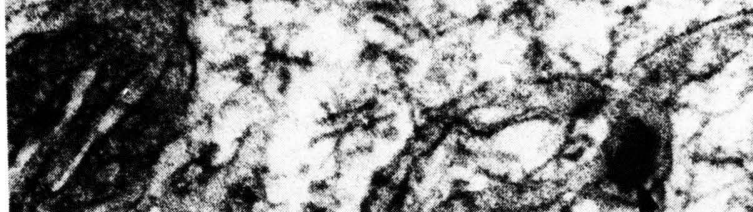

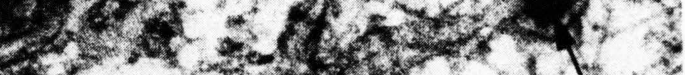

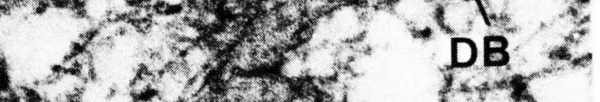

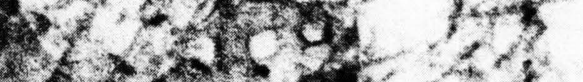

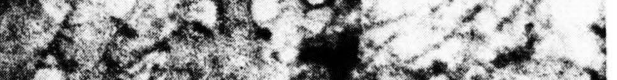

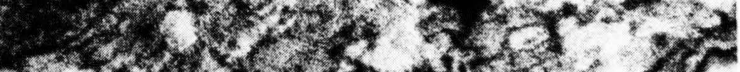

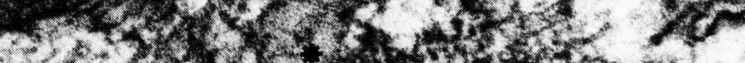

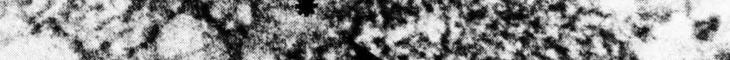

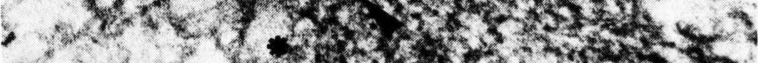

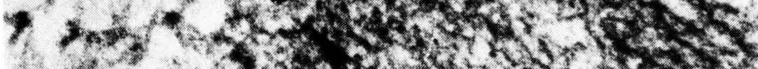

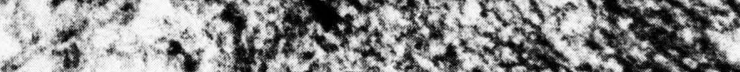

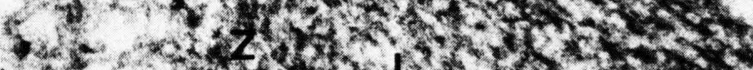

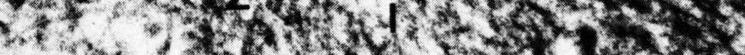

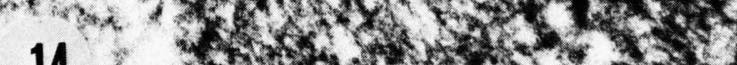
14.3 and

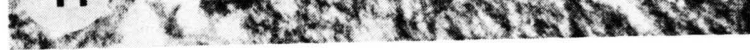
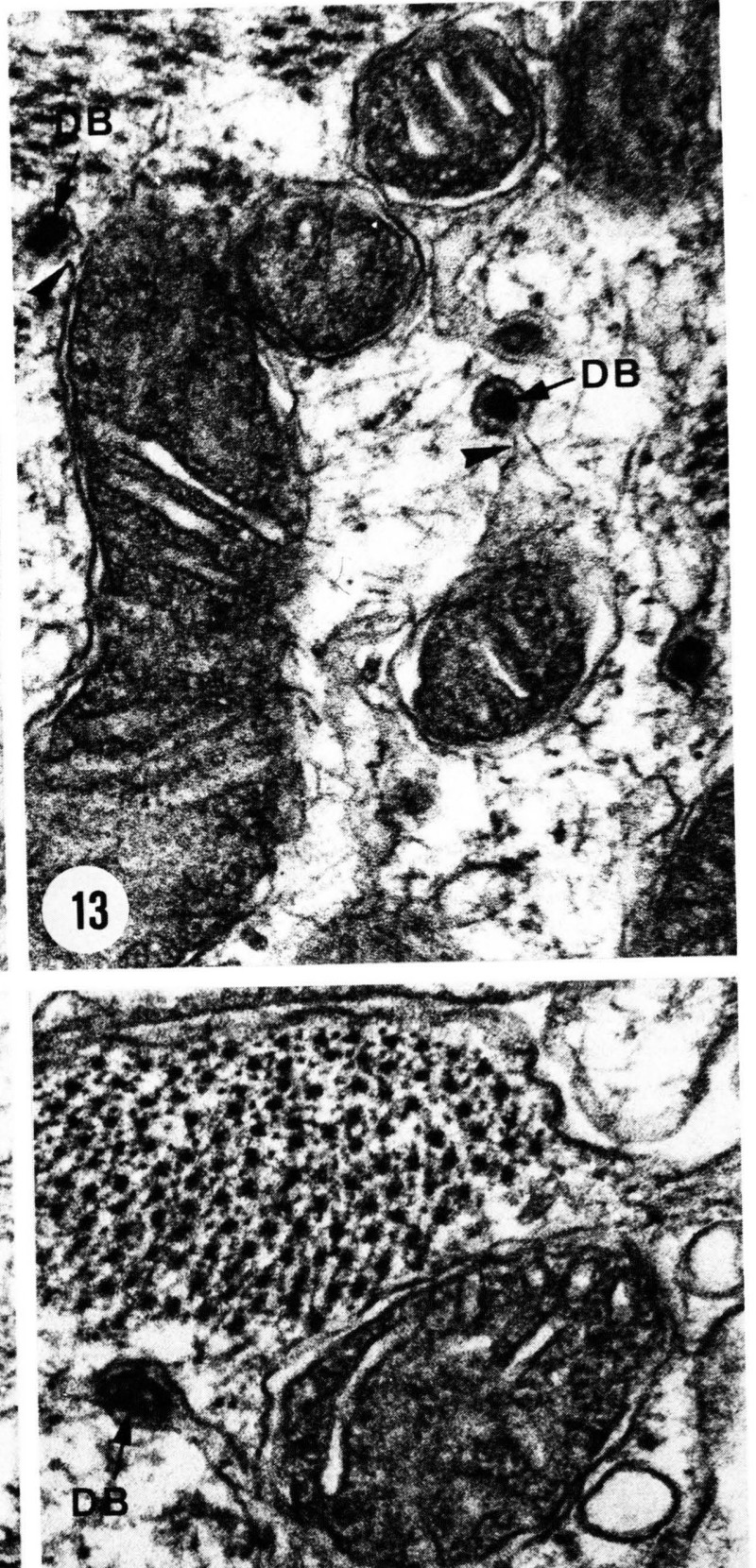
W d.

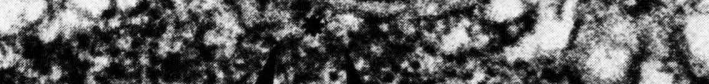

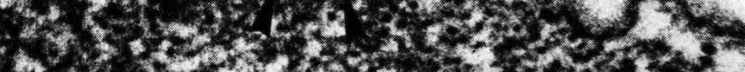

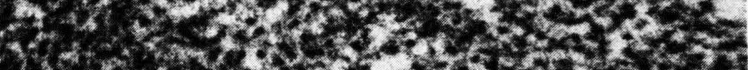

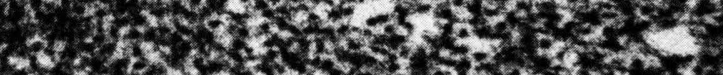

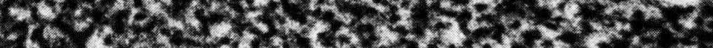

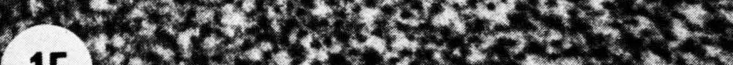

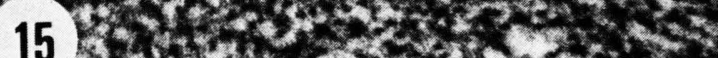

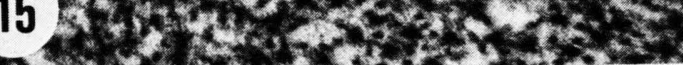



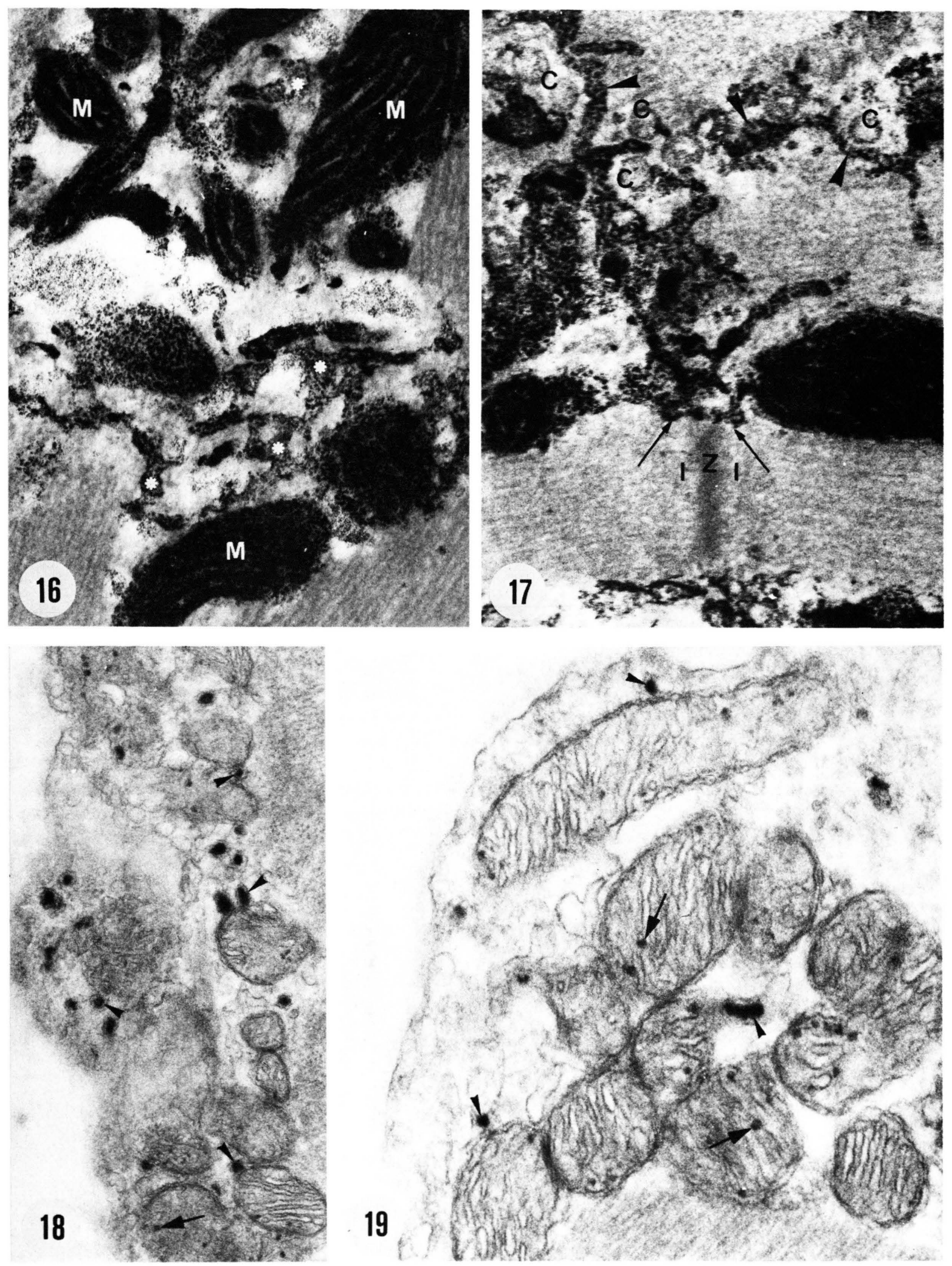


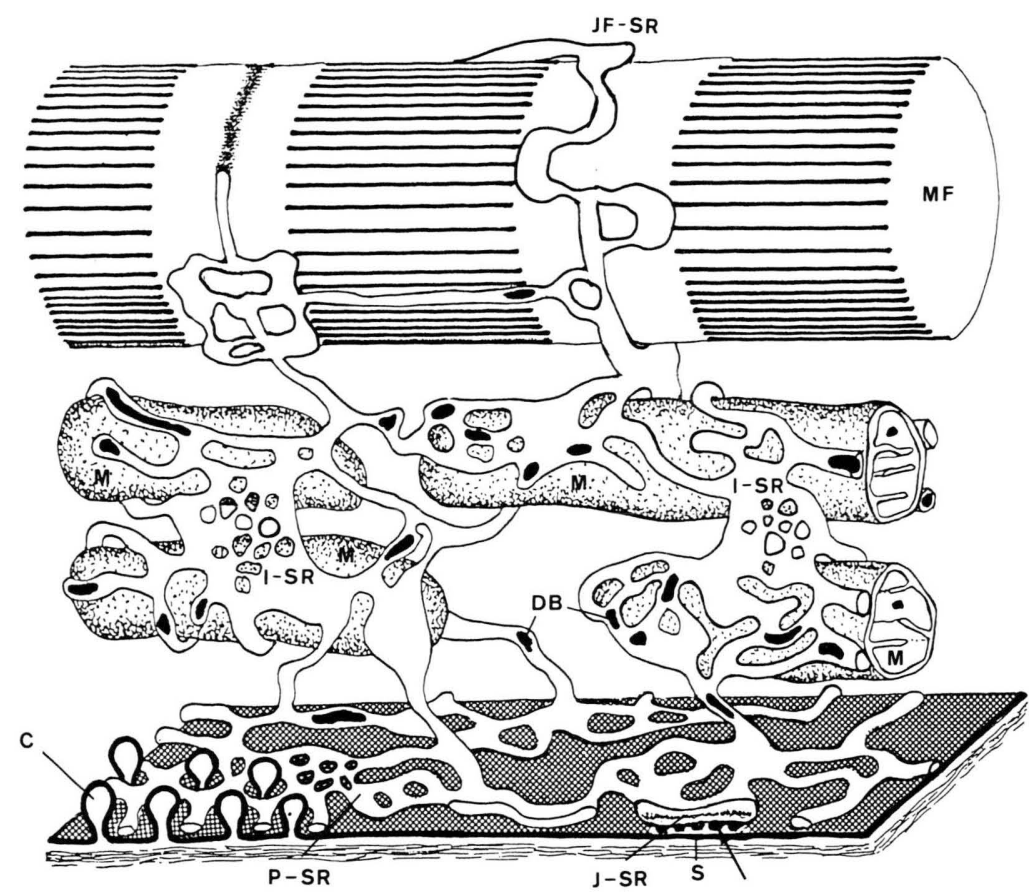

Fig. 20. Schematic drawing of the whole sarcoplasmic reticulum illustrating its three different regions and relations they respectively contract with different cellular organelles:

- peripheral SR, beneath the sarcolemma (S) with two components: “junctional SR" (J. SR) which performs “couplings" with the sarcolemma (arrows) and peripheral SR s. str. (P. SR) which is often intermingled with the caveolae (C).

- juxtafibrillar SR (JF. SR) closely related to myofibrils (MF) at the level of Z line and I band.

- intermediary SR (I. SR) containing dense bodies (DB) which performs the junction between the two preceding regions and also shows close topographical relations with the mitochondria (M).

sely sectioned and unstained. The juxtafibrillar SR can also be clearly observed, at the level of $Z$ lines and of I bands (Fig. 17).

\section{Determination of sites able to bind calcium}

Replacement of calcium by strontium, followed by an osmic fixation gives sections where, without any further staining, the only electron dense structures are mostly intra-mitochondrial granules and dense bodies of the SR (Figs 18 and 19). These electron micrographs also confirm that the dense bodies of the SR are preferentially localized in the vicinity of the mitochondria.

\section{Discussion}

The SR of the sino-auricular fibres of frog heart appears as a complex structure linking together and giving unity to three regions topographically and morphologically clearly defined: on the one side, sarcolemma bound "peripheral SR", particularly the "junctional SR" (couplings) and on the other, "juxtafibrillar SR", both being linked together by "intermediary SR". The latter is frequently formed into fenestrated saccules and shows close topographical relations with mitochondria (Fig. 20).

+ These results illustrate the large development of the SR of the sino-auricular fibres and do not agree

Figs 16, 17. Metallic impregnation after the technique of Thiéry and Rambourg. The peripheral sarcoplasmic reticulum (arrowheads) is intermingled with the caveolae $(\mathrm{C})$. The intermediary sarcoplasmic reticulum (*) is well extended and shows close topographical relations with the mitochondria (M). The juxtafibrillar sarcoplasmic reticulum (arrows) is in close relationship with the myofibrils (at the level of $\mathrm{Z}$ line and I band). $0,5 \mu \mathrm{m}$ section.

Fig. 16. $\times 74000$; Fig. 17. $\times 76000$.

Figs 18, 19. Demonstration of sites able to bind strontium (and calcium) after the technique of Peachey. Unstained sections. Only intra-mitochondrial granules (arrows) and intra-reticular dense bodies (arrowheads) are marked.

Fig. 18. $\times 44000$; Fig. 19. $\times 62000$. 
with those authors who have studied the ultrastructure of frog heart auricle [27] or ventricle [18, 19, 22]. On the contrary, they confirm and amplify the conclusions of Masson-Pévet [28] who insisted on the relatively great development of the SR in frog auricle. As Rybicka [29] pointed out, technical difficulties of preservation of the SR in cytoplasmic zones are probably responsible for its frequent underestimation in certain types of material rich in cytoplasm (frog heart, conducting tissue in mammals, ...) and of the accompanying absence of microtubules and microfilaments. Nevertheless, the development of the SR of frog heart sino-auricular fibres is important enough to justify a functional role. Its various differentiations and its relations with the mitochondria reinforce this conclusion.

"Couplings" have already been mentioned in frog auricle by Denoit-Mazet and Vassort [27] and by Masson-Pévet [28]. The peripheral SR with its two aspects (junctional SR and tubules reticulum) has been well described by Forbes et al. [44] in mouse ventricle and mostly in lizzard ventricle (Anolis carolinensis). A network of SR tubules lying close to the caveolae, comparable to the peripheral SR s. str. of the frog sino-auricular fibres, has also been described by Gabella [31] in the rat myocardium. Intra-reticular dense bodies have already been observed by Nayler and Merrillees [17] in toad ventricle, by Sommer and Johnson [19] and by Page and Niedergerke [22] in frog ventricle. In the latter material, Winegrad [32] demonstrated that the dense bodies show a positive reaction after partial replacement of calcium by strontium followed by osmic fixation. An excellent description of the juxtafibrillar SR has been given by Page and Niedergerke [22] in frog ventricle. Finally Forbes and Sperelakis [30] mentioned the superposition of SR and mitochondria in mouse ventricle which suggests an interconnection between these two elements.

+ Topographical relations between intermediary SR and mitochondria support the view of some functional link between these two organelles; the first hypothesis to be formulated in muscle tissue is that this link could concern some role of the mitochondria in the movements of calcium. It can be recalled that intra-reticular dense bodies which seem to be able to bind strontium (and calcium) are especially abundant in the juxta-mitochondrial SR. It would also be recalled that Diculescu, Popescu and Ionescu [33] and Diculescu, Popescu, Ionescu and
Butucescu [34] who used the oxalate method for the cytochemical visualization of calcium demonstrated in rat ventricle, the occurrence of a juxta-mitochondrial SR, particularly rich in calcic deposits. In this hypothesis, ultrastructural data would support those biochemical results which favour a participation of the mitochondria in the calcium movements during excitation-contraction coupling. In addition, they suggest that mitochondria could play their role by being coupled to the SR, without however eliminating the possibility of an eventual direct calcium ex change with the sarcoplasm. It is interesting to note that a new possibility to preserve and to quantitate intracellular compartmentalization of diffusible ions is given by a method recently described by Somlyo et al. [35] on frog skeletal muscle fibres.

The actual nature of relations between SR and mitochondria cannot be documented for the moment. We already pointed out that the apparent continuity between the external mitochondrial membrane and the SR membrane may well be artifactual, due to the obliquity of the section plans. One can imagine at least two types of connection between these organelles, some inter-membranar junctional structure on the one side, actual fusion - possibly a dynamic phenomenon - of membranes on the other side.

These SR-mitochondria relations may be placed in the more general case of relations between endoplasmic reticulum (ER) and mitochondria. Such relations seem to occur widely in various tissues. For example, relations of this type have been described by Osborne [36] in the terminal motor axon of fibres innervating parietal musculature of Carausius morosus, by Taxi [37] in sympathetic neurons of the frog, by Russell and Burguet [38] in the Leydig cells of rat testis and Thiéry et al. [39] in the cells of the convoluted proximal tubule of rat nephron. In the two last examples, pictures of ER forming flattened fenestrated saccules, closely apposed to mitochondria, very similar to those in the sino-auricular fibres of the frog, have been described. Meier et al. [40] have also demonstrated in rat liver, an association of granular ER-mitochondria, the integrity of which is necessary for the assembly of the cytochrome P 450. In this example, inter-membranar junctional structures have been observed.

+ The division of the SR in 3 differentiated regions is interesting by its functional implications. From this point of view, and taking into account the 
information presently available on the subject, the following hypothesis can be presented:

- The junctional SR, directly coupled to the sarcolemma and triggered by the muscular action potential could release activator calcium into the hyaloplasm. Indeed junctional SR is homologous to the terminal cisternae of the SR which acts in this way in skeletal striated muscle fibres [41].

- The juxta-fibrillar SR could pump the calcium out of the myofibrils. It is only a small fraction of the whole SR of the cell, and this could explain the low pumping activity of the SR of frog myocardium, pointed out by most physiologists [42].

- The intermediary SR could assume 1) the communication between the two former regions of the SR, permitting closure of the intracellular circuit of calcium 2) the coupling with the mitochondria which could constitute a lateral calcium compartement able to act in regulating the circuit.

It is interesting to note that already Chapman and Léoty [43] have also concluded on purely physiologi-

[1] B. Chance, J. Biol. Chem. 240, 2729 - 2748 (1965).

[2] A. L. Lehninger, Biochem. J. 119, $129-138$ (1970).

[3] E. Carafoli and M. Crompton, Ann. N. Y. Acad. Sci. 307, 269 - 284 (1978).

[4] E. Carafoli, J. Mol. Cell. Cardiol. 7, 83 - 89 (1975).

[5] W. E. Jacobus, R. Tiozzo, G. Lugli, A. L. Lehninger, and E. Carafoli, J. Biol. Chem. 250, $7863-7870$ (1975).

[6] H. Affolter, M. Chiesi, R. Dabrowska, and E. Carafoli, Eur. J. Biochem. 67, $389-396$ (1976).

[7] N. Haugaard, E. S. Haugaard, N. H. Lee, and R. S. Horn, Fed. Proc. 28, 1657 - 1662 (1969).

[8] R. S. Horn, A. Fyhn, and N. Haugaard, Biochim. Biophys. Acta 226, $459-470$ (1971).

[9] A. Scarpa and P. Graziotti, J. Gen. Physiol. 62, $756-772$ (1973).

[10] A. Vinogradov and A. Scarpa, J. Biol. Chem. 248, $5527-5531$ (1973).

[11] T. Kitazawa, J. Biochem. 80, 1129 - 1147 (1976).

[12] S. Schaffer, B. Safer, and J. R. Williamson, FEBS Letters 23, $125-130$ (1972).

[13] S. Bloom, A. J. Brady, and G. A. Langer, J. Mol. Cell. Cardiol. 6, 137 - 147 (1974).

[14] J. Tsokos, R. Sans, and S. Bloom, Life Sciences 20, $1913-1922$ (1977).

[15] C. Léoty and G. Raymond, Pflügers Arch. 334, $114-128$ (1972).

[16] G. Vassort and O. Rougier, Pflügers Arch. 331, $191-203$ (1972).

[17] W. G. Nayler and N. C. R. Merrillees, J. Cell Biol. 22, 533 - 550 (1964).

[18] N. A. Staley and E. S. Benson, J. Cell Biol. 38, $99-114$ (1968).

[19] J. R. Sommer and E. A. Johnson, Z. Zellforsch. 98, $437-468$ (1969).

[20] R. A. Chapman, J. Physiol. 215, 48 - 49 P (1971).

[21] M. Masson and D. Gros, J. Microscopie 11, 78 (1971).

[22] S. G. Page and R. Niedergerke, J. Cell Sci. 11, $179-203$ (1972). cal grounds (study of caffeine contractures), that in the heart of several species of mammals, occur a closed system of four intracellular calcium compartements (three in the SR and one in the hyaloplasm) and a side calcium compartment probably mitochondrial in nature.

This scheme only deals with activator calcium of intracellular origin, responsible of the tonic component of the contraction and discards activator calcium of extracellular origin, responsible of the phasic component of the concentration. In physiological conditions both circuits of calcium cannot be separated.

\section{Acknowledgements}

This work was supported by a DGRST programme grant (Biologie et fonction du myocarde, contrat $\mathrm{n}^{\circ}$ 75.7.0313). The authors wish to acknowledge the excellent technical assistance of Mrs. Christiane Salat.

[23] F. Mazet, J. Microscopie Biol. Cell. 24, $117-122$ (1975).

[24] M. J. Karnovsky, J. Cell Biol. 27, 137 A (1965).

[25] G. Thiéry and A. Rambourg, J. Microscopie Biol. Cell. 26, 103 - 106 (1976).

[26] L. D. Peachey, J. Cell Biol. 20,95 - 111 (1964).

[27] F. Denoit-Mazet and G. Vassort, J. Microscopie 12, $413-424$ (1971).

[28] M. Masson-Pévet, Thèse $3^{\circ}$ Cycle, Poitiers (1972).

[29] K. Rybicka, Anat. Rec. 189, 237 - 262 (1977).

[30] M. S. Forbes and N. Sperelakis, J. Ultrastructure Res. 58, 50 - 65 (1977).

[31] G. Gabella, J. Ultrastructure Res. 65, 135-147 (1978).

[32] S. Winegrad, J. Gen. Physiol. 62, 693 - 706 (1973).

[33] I. Diculescu, L. M. Popescu, and N. Ionescu, Exp. Cell Res. 68, 210-214 (1971).

[34] I. Diculescu, L. M. Popescu, N. Ionescu, and N. Butucescu, Z. Zellforsch. 121, 181 - 198 (1971).

[35] A. V. Somlyo, H. Shuman, and A. P. Somlyo, J. Cell Biol. 74, 828 - 857 (1977).

[36] M. P. Osborne, Insect Ultrastructure, vol. 5, pp. 77 - 100, Blackwell, Oxford 1970.

[37] J. Taxi, Frog Neurobiology, pp. $93-150$. Springer, Berlin-Heidelberg 1976.

[38] L. Russell and S. Burguet, Tissue and Cell 9, 751 - 766 (1977).

[39] G. Thiéry, D. Guérette, J. Forget, and M. Bergeron, Biol. Cell. 32, 11 a (1978).

[40] P. J. Meier, M. A. Spycher, and U. A. Meyer, Exp. Cell Res. 111, $479-483$ (1978).

[41] M. Endo, Physiol. Rev. 57, 71 - 108 (1977).

[42] R. A. Chapman and D. J. Miller, J. Physiol. 242, $589-613$ (1974).

[43] R. A. Chapman and C. Léoty, J. Physiol. 256, $287-314$ (1976).

[44] M. S. Forbes, B. A. Plantholt, and N. Sperelakis, J. Ultrastructure Res. 60,306 - 327 (1977). 